\title{
Investigating Relationships: Thoughts on the Pitfalls and Directions
}

\author{
JEFFREY W. BLOOM \\ Northern Arizona University (USA)
}

The feature article for this issue, entitled "(Re)Imagining Teacher Preparation for Conjoint Democratic Inquiry in Complex Classroom Ecologies," begins to carve out one aspect of the importance of relationship in the context of schooling. For the most part, the institution of schooling has ignored relationship. Instead, blaming teachers and students has become the modus operandi. Zero tolerance, accountability, among the many other sound bites in the politics of education categorically ignore the significance of relationship and its critical role not only in student-teacher dynamics, but also in all aspects of learning and personal growth. In fact, within the current political context, relationship is missing from the equation. Scripted curricula and requirements that teachers sign allegiance to these curricula, intensive teaching to the tests, the push for strict and specific national standards, and the desire to have all students working on the same "thing" (and not relationships of any kind!) at the same time, are all tremendous obstacles to developing interpersonal relationships in the classroom and to learning relationships. Of course, the notion of relationships is much more extensive than just those connections that exist between people. Relationships should be the material of what we learn and teach (Bateson, 1979/2002; Donaldson, 1992). Since we are interested in complex systems, we need to see relationships as the material of systems, as well as to see relationships as systems themselves.

Although I believe the major focus of this article is of great importance, I do have some concerns. The authors have brought to bear a number of different paradigmatic approaches in crafting this research and the resulting article. In many cases, such a mix of paradigms can provide some intriguing insights. However, I found this paradigmatic mix problematic in this article. Complexity theories are, by definition, at opposition to 
positivistic, reductionist, and mechanistic paradigms (Capra, 1996). The concerns and assumptions of complexitivists are quite different from those of positivists. In fact, in the context of social sciences research, positivist perspectives have led us down a garden path wrought with briar patches. By trying to quantify and find predictability in human behavior and by focusing on behavior rather than cognition, emotion, etc., researchers have missed the richness and complexity of human beings. The seduction of positivism is powerful and insidious. Our desires to create neat and orderly categories for everything is comforting in a world of unpredictability and seeming chaos. Even though we may align ourselves with a complexitivist worldview, the comforts of positivistic approaches may seep into and undermine our attempts to understand particular phenomena. I fear this paper suffers from just such a conflict in paradigms. Simple dichotomies, such as the "autonomy-supporting vs. controlling" continuum, appear to be intriguing, but can be misleading. Have the complexity of teacher-student relationships been reduced to a simple binary of behaviors? I don't think so. Gregory Bateson, on whom the authors have based much of their work, saw relationships as complex systems embedded in a variety of contexts, including culture. In fact, Bateson (1972/2000; 1979/2002; 1991) asserted that context is absolutely essential for understanding relationships. To a great extent, context and culture are absent from the article. Part of the reason why context is so important is that it is just this context (and culture) that sets up what Bateson (1991) refers to as "rules of contingency" in relationships. From a somewhat different perspective, Kelso and Engstrøm (2006) have been examining complementary pairs or binary relationships. The essential aspect of such pairings or relationships is their "coordination dynamics." In other words, the major concern in trying to understand relationships involves the dynamics of how relationships and their rules of contingency are coordinated, which, for the most part, are embedded and determined by context. We can see how these rules of contingency appear in the data of the present article, but these contingencies or coordination dynamics are not examined in depth. The expectations of students and teachers arise from these contingencies, but without an examination of the contexts of each student, the classrooms, the schools, the communities, and the teachers, we are left with only a glimpse of possibilities and very little understanding of the full extent and depth of the relationships. In addition, the authors' claims about the differing "views" of relationship between teachers and students are supported neither by descriptions of context, nor by the what Bateson (1979/2002) suggests as the "double description" which is always the "relationship." This double description, which frequently is not described through language, can provide insights into the contingencies and coordination dynamics. In order to understand this double description we need a variety of methodologies, such as a variety of observational techniques, interviews, and ways at describing the dynamic interconnectedness of contexts, culture, rules of contingencies, relationships, and other aspects of relational systems.

The almost exclusive use of survey or questionnaire data is problematic on several fronts. People lie, although not always intentionally. Although this statement has been popularized on the TV show "House," Bateson (1979/2002) would agree: “...we project 
our opinions of self onto the outer world, and often we can be wrong about the self..." (p. 127). In market research, Bertrand and Mullainathan (2001) have come to the same conclusion that people lie in responding to surveys and questionnaires. Part of this problem may be that we think we believe in something and act consistently with these beliefs, but we rarely do. Such data also tend to be fairly superficial. They do not elucidate deeper patterns. Questionnaires also reflect the biases of the researchers. The questions asked are formulated based upon specific theoretical frameworks, concerns, and biases. If answer choices are limited, the bias problem is compounded even further. To rely entirely of questionnaire data is of questionable value. Observations of actions in the classroom would have been very helpful in developing more in-depth descriptions of the dynamics of the relationships between teachers and students and the enactment of one's beliefs and values. Interviews also could have helped to explore conflicts in beliefs and actions, and so forth. So, making claims such as, "it appears that children did not simply see what they expected to see, but rather, actually elicited in their teachers' behavior [sic] the very styles they had anticipated" are extremely tenuous, at best. There is a "suggestion" that this elicitation occurs, but there are not enough data to support this claim.

This article also embraces the use of different paradigms in the research it describes. However, the use of conflicting paradigms in research may be quite problematic. Not only are the conflicting assumptions between paradigms an issue, but also the potential for the assumptions of one paradigm undermining the premises of the other are increased. Catherine Bateson and her father (1987/2005) suggested that our language (especially English and other European languages) is concerned mostly with "things" and not with relationships. Then, of course, when focusing on "things," we tend to use positivistic paradigms that focus on quantification. For Bateson (1979/2002), “... quantity and pattern are of different logical type and do not readily fit together in the same thinking" (p.49). Relationships are not measureable or quantifiable. They are patterns. Bateson (1981) takes this problem with quantification a step further in suggesting that,

... we have to ask about change of patterns, change of very fundamental settings in our society. And we approach that... in a state of mind totally unequipped to deal with it, namely a state of mind which is thinking all the time on the quantitative side.... (p. 353)

Harries-Jones (1995/2002) describes Bateson's problem with quantification, where quantifying is concerned with "sameness" and description is concerned with "difference." It is this "difference" that is essential for understanding relationship. If we do not focus on difference, relationship, and description in research on the interconnectedness of relationship, culture, and context, then our understandings of this interconnectedness either is going to come up significantly short or is going to fail to provide significant understandings.

So, the reliance on language to investigate relationships and then quantifying this language is like double jeopardy. First, using only language as a way of "getting at" an understanding of relationship is problematic. Then, the problem is further compounded by quantifying. Language is not sufficient in itself to describe relationship. The quantification is not about relationship, but about the "things" that may be in 
relationship. And, quantification does not describe relationship, at least, not human relationships.

This notion of conflicting assumptions is apparent in the discussion of "oppositional defiant disorder" almost midway into this article. The very labeling of a "disorder" is based on very different assumptions from what one may have if adopting a "relationships view." From a positivistic view, we can label a "condition" that resides in an individual as a disorder. However, from a view based on relationships, rather than on separate and individual entities, a "disorder" does not reside in the individual. Rather, the "disorder" is a pathology in the dynamics of the relationship not in the individuals. Yet, we use the language of individual entity or positivism to describe the situation and what actions need to be taken. An "intervention" is directed at the individual, not the relationship. "Time-outs" are for an individual. "De-escalation" is directed at the individual or individuals. If we truly want to take a relationship view, we need a new language based on very different sets of assumptions.

Other evidence of conflicting assumptions found in the paper includes the notion of "rubrics," which are clear attempts to demarcate, separate, and solidify. They also set up the basis for attempts at "measuring." In this paper, these attempts are focused on solidifying and measuring student cognition or "levels of reflection." As the authors mention in their discussion of Martin Buber's work, relationships are characterized by "Thou" and not "It." However, the whole notion of rubrics, measurement, and quantifying human beings is geared toward creating "It." The insidious nature of positivism also has crept into the authors' use of Carl Rogers' approaches to communication. Although Rogers work pre-dated the complexity sciences (but not cybernetics as the forerunner of complexity sciences), as such, his work was antithetical to the positivist-behaviorist work that had been dominant in the mid-twentieth century. The attempt to make "purposeful" the communication techniques has the effect of creating a more solidified, "sequentialized," and linear approach to communication as opposed to communication that is a nonlinear, natural part of relationship.

Those of us interested in continuing the research into relationships need to keep in mind a number of points.

- Relationship cannot be understood separate from context, including culture.

- Relationships cannot be quantified or measured.

- Relationships are complex systems.

- We need to avoid the seduction of positivism.

- We need to be more rigorous in avoiding conflicting assumptions and misleading use of language.

- Some important questions for our research include:

- How are teacher-student relationships negotiated and coordinated?

- How can teachers work on developing good relationships with students and among students?

- How do pathological or dysfunctional relationships arise in classrooms and schools? 
Bateson's (1972/2000; 1991) notion of double bind may be an important concept for examining the creation of pathology in relationships. Double binds are not just "damned if you do, damned if you don't," but are also deeply embedded within the context of relationships. The context in such situations tends to intensify the pathological patterns of relationship.

- How can pathological relationships be reworked?

- What are the contexts of teacher-student relationships? How are they characterized? How do they affect relationships?

- How can we help pre-service and in-service teachers understand the nature of relationships? How can we help them focus on relationships in the classroom?

- What effect does a focus on relationships have on teachers, students, and classrooms?

\section{References}

Bateson, Gregory. Steps to an ecology of mind. Chicago: University of Chicago Press, 1972/2000.

Bateson, Gregory. Mind and nature: A necessary unity. Cresskill, NJ: Hampton Press, 1979/2002.

Bateson, Gregory. "Paradigmatic conservatism," in Rigor and imagination: Essays from the legacy of Gregory Bateson, edited by Carol Wilder \& John H. Weakland, 347-355. New York: Praeger, 1981.

Bateson, Gregory. Sacred unity: Further steps to an ecology of mind (R. E. Donaldson, Ed.). New York: Harper Collins, 1991.

Bateson, Gregory, and Mary Catherine Bateson. Angels fear: Towards an epistemology of the sacred. Cresskill, NJ: Hampton Press, 1987/2005.

Bertrand, Marianne, and Sendhil Mullainathan. "Do people mean what they say? Implications for subjective survey data." The American Economic Review 91, no. 2, (2001): 67-72.

Capra, Fritjof. The web of life: A new scientific understanding of living systems. NY: Anchor/Doubleday, 1996.

Donaldson, Margaret. Human minds: An exploration. New York: Allen Lane/Penguin, 1992.

Harries-Jones, Peter. Recursive visions: Ecological understanding and Gregory Bateson. Toronto, Ontario: University of Toronto Press, 1995/2002.

Kelso, J. A. Scott, and David A. Engstrøm. The complementary nature. Cambridge, MA: MIT Press, 2006.

\section{Acknowledgements}

I'd like to thank Elizabeth Miller-Jensen for her insightful comments on this article and for her feedback on this manuscript.

\section{About the Author}

Jeffrey W. Bloom, Ed.D., is professor of elementary science education in the Department of Teaching and Learning, College of Education, Northern Arizona University. His research interests involve (a) teaching and learning as complex systems with special emphasis on recursive approaches to highly transferrable learning, (b) classroom communities as complex systems, (c) metapatterns as analytical and design tools and as conceptual content for learning, and (d) the implications of Gregory Bateson's work on teaching, learning, and curriculum. He can be contacted at jeff.bloom@nau.edu or jeff@jeffbloom.net. 
(c) Copyright 2011. The author, Jeffrey W. Bloom, assign to the University of Alberta and other educational and non-profit institutions a non-exclusive license to use this document for personal use and in courses of instruction provided that the article is used in full and this copyright statement is reproduced. The author also grants a non-exclusive license to the University of Alberta to publish this document in full on the World Wide Web, and for the document to be published on mirrors on the World Wide Web. Any other usage is prohibited without the express permission of the authors. 